\title{
A Rare Case Report: Imaging Features of Cystic encephalomalacia of Infancy following Antenatally Detected Intraparenchymal and Intraventricular Hemorrhage
}

\author{
Dipti A. Shah ${ }^{1}$, Vaishali Sanghrajka ${ }^{2}$ \\ ${ }^{1}$ Professor \& Head of Department, Department of Radiodiagnosis, AMC MET Medical College, Sheth L.G.Hospital, Maninagar, \\ Ahmedabad, ${ }^{2} 3$ rd year Resident doctor, Department of Radiodiagnosis, AMC MET Medical College, Sheth L.G.Hospital, \\ Maninagar, Ahmedabad, India
}

Corresponding author: Sanghrajka Vaishali Jiteshbhai, A-104, Shivalaya-3, O/p Sachin Tower, Shyamal 100ft Road, Satellite, Ahmedabad, Gujarat-380015, India

DOI: http://dx.doi.org/10.21276/ijcmsr.2019.4.4.25

How to cite this article: Dipti A. Shah, Vaishali Sanghrajka. A rare case report: imaging features of cystic encephalomalacia of infancy following antenatally detected intraparenchymal and intraventricular hemorrhage. International Journal of Contemporary Medicine Surgery and Radiology. 2019;4(4):D103-D106.

\section{A B S T R A C T}

Introduction: Intracranial hemorrhage is a common event in premature infants. It may occur in utero also, and prenatal diagnosis by either ultrasound or magnetic resonance imaging (MRI) has been reported. Hemorrhage may occur either within the cerebral ventricles, cerebral parenchyma or subdural space.

Case report: Here with we report a case of an infant who was diagnosed as having intraparenchymal and intraventricular hemorrhage by antenatal ultrasound and MRI at 32 weeks of gestation. The infant developed cystic encephalomalacia changes later at about 1 month of life. In the following case report, imaging findings of intracranial hemorrhage and cystic encephalomalacia in an infant is described.

Conclusion: Cranial ultrasound is diagnostic as well as safe for follow up of the patient. MRI should also be considered in assessment of fetal ICH as detailed informative and confirmative modality

Keywords: Ultrasound, Magnetic Resonance Imaging, Intraparenchymal and Intraventricular Hemorrhage, Cystic Encephalomalacia

\section{INTRODUCTION}

Intracranial hemorrhage $(\mathrm{ICH})$ is a common event in premature infants. Factors predisposing to in utero $\mathrm{ICH}$ include a variety of conditions, mostly maternal trauma and fetal coagulation disorders. In many cases, however, the cause is not identified. The incidence is unclear, although an estimate of 1 in 10,000 pregnancies have been suggested.

Ultrasound findings of fetal $\mathrm{ICH}$ are variable and at times subtle. In general, this condition is considered difficult both to identify and to differentiate from other intracranial lesions. The most common abnormality found in case of fetal $\mathrm{ICH}$ on ultrasound is ventricular enlargement. Other features on ultrasound suggestive of fetal $\mathrm{ICH}$ are loss of normal cerebral landmarks, avascular intracranial mass lesion with variable echogenicity and increased periventricular echogenicity. Blood clot change in echogenicity over time and undergo eventual resorption. ${ }^{1}$

Fetal ICH is rare but important diagnosis to make in utero, as it has prognostic implications for the current and potentially for future pregnancies. Ultrasound diagnosis of fetal ICH can be difficult particularly in late gestation (when it is most likely to occur), with multiple pregnancies or with maternal obesity. Fetal MRI can overcome the acoustic access issues caused by overlapping of fetal parts, challenging fetal position and ossified skull vault

Cystic encephalomalacia is a rare cerebral disorder seen predominantly in premature neonates where cerebral parenchyma is replaced by irregular cystic areas of variable sizes which is the final result of diffuse brain insult in the late gestation, during or after birth. The condition can be caused by intracerebral hemorrhage, asphyxia, intracranial infection. Perinatal cerebrovascular accident is believed to be the most common cause. Cystic encephalomalacia is a condition in which portions of brain are replaced by numerous loculated cysts in the white matter and cortex. Usually, the lesions are seen in the vascular territory of anterior cerebral artery and middle cerebral artery with sparing of temporal lobes, thalami, deep gray matter and posterior fossa., 2,3,4

\section{CASE REPORT}

A $26 \mathrm{yr}$ old fourth gravida patient known case of sickle cell disease presented at 32 weeks of gestation with bleeding per vaginum to obstetrics and gynaecology department, and AMC MET Medical College \& Sheth L.G. Hospital and patient was referred to the department of radiodiagnosis, AMC MET Medical College \& Sheth L.G. Hospital for antenatal ultrasound scan. 

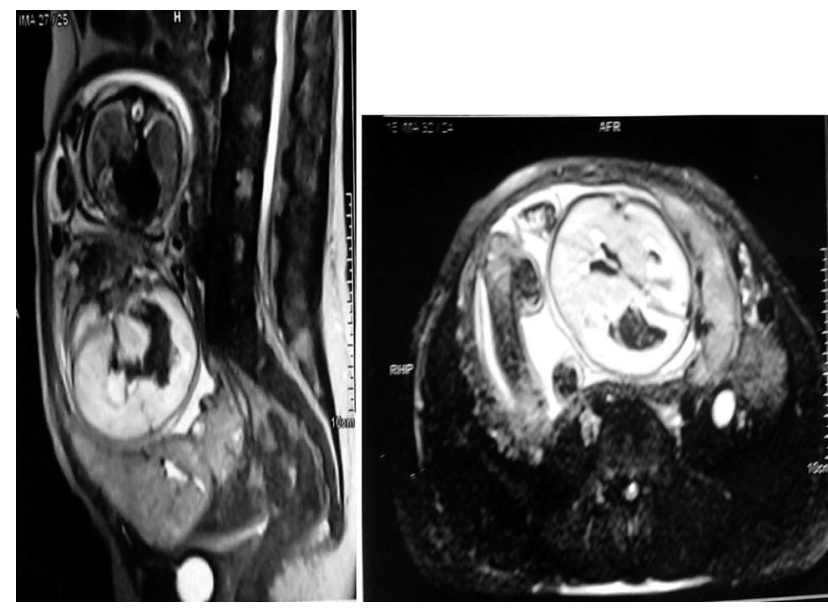

Figure-1: Antenatal MRI of abdomen and pelvis - Sagittal and Axial T2 weighted images showing hypointense area in left frontoparietal region, suggestive of intraparenchymal hemorrhage with dilated ventricles and hypointense content on T2 weighted images involving third, fourth and both lateral ventricles of fetal brain, suggestive of intraventricular hemorrhage.

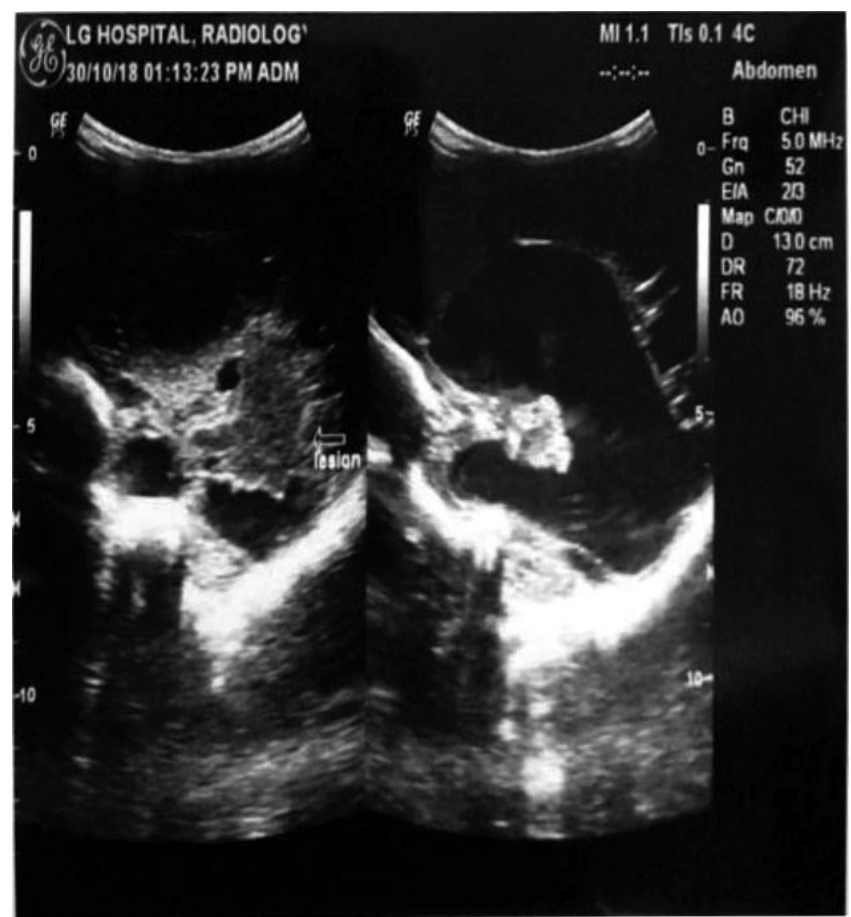

Figure-2: Coronal and sagittal ultrasound image of brain through anterior fontanelle- 26 x $16 \mathrm{~mm}$ lesion with heterogenous echogenicity in left frontoparietal and basal ganglia, suggestive of chronic intraparenchymal hemorrhage with dilatation of bilateral lateral ventricles.

Patient had history of previous three abortions (still births). No family history of genetic or congenital anomalies was found. There was no history of trauma or no history that suggested infection or exposure to toxins. No previous antenatal ultrasound scans were done.

Antenatal ultrasound findings showed hyperchoic lesion in left frontoparietal region suggestive of intraparenchymal hemorrhage with heterogenous predominantly hyperechoic lesion in ventricles suggestive of intraventricular hemorrhage

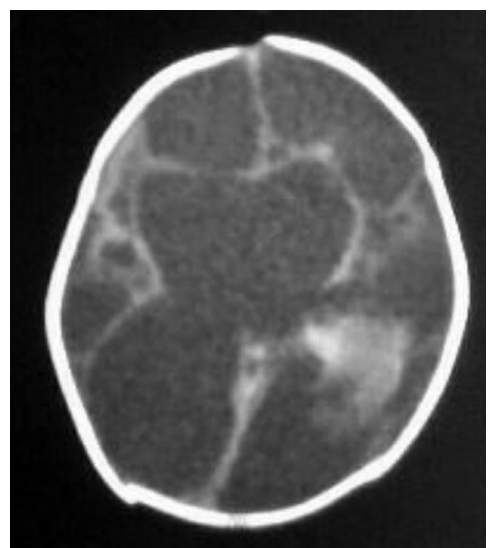

Figure-3: Axial NCCT Brain- Severe loss of cerebral cortex with cerebral hemisphere replaced by multiple cystic encephalomalacia changes. Approx. 25x16 mm heterogenous density area in left frontoparietal and basal ganglia region, suggestive of chronic hemorrhage with dilatation of both lateral ventricles.
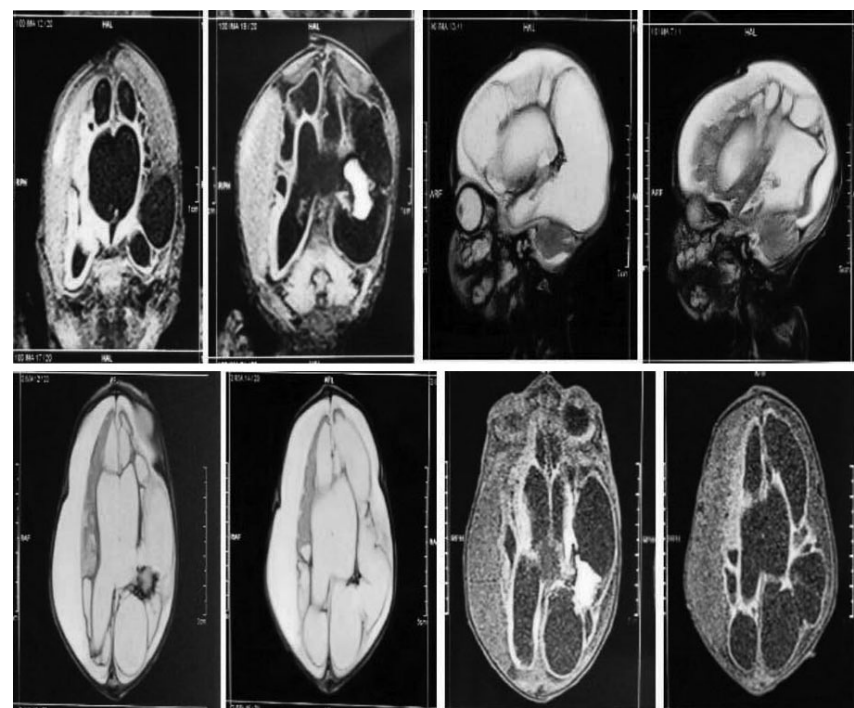

Figure-4: MRI Brain- Coronal FLAIR, Sagittal and axial T2 and axial T1 weighted images respectively showing Cystic encephalomalacia changes in the form of multiple large hypointense areas on T1W and hyperintense areas on T2W images noted involving bilateral fronto-parietooccipito-temporal regions with hyperintense area involving left frontoparietal region on T1W images, suggestive of subacute hemorrhage.

in fetal brain. Placenta previa was also present. Antenatal ultrasound findings were confirmed by MRI scan of mother next day after ultrasound.

MRI of abdomen with pelvis of mother was performed and T1 and T2 weighted serial sections obtained in sagittal, axial and coronal planes using body coil and respiratory compensation on a 1.5 Tesla scanner.

MRI findings were also suggestive of hyperintense area on T1 weighted image in left frontoparietal region, suggestive of intraparenchymal hemorrhage with dilated ventricles and hypointense content on T2 weighted images involving third, fourth and both lateral ventricles of fetal brain, suggestive of intraventricular hemorrhage.(Figure 1) 
A preterm baby was delievered by cesarian section at 32 weeks of gestation with APGAR score 04 at 1 minute and 06 at 5 minute, weight $1.7 \mathrm{~kg}$ and head circumference of $32 \mathrm{~cm}$ and not cried immediately after birth so required resuscitation in the form of bag and mask ventilation and kept on c-PAP for 1day.

Cranial ultrasound of newborn baby was performed through anterior fontanelle approach with both coronal and sagittal images obtained of the ventricles, entire brain including surface features, which revealed dilatation of bilateral lateral ventricles with hyperechoic foci within, suggestive of intraventricular hemorrhage. Focal lesion with heterogenous echogenicity noted in left frontoparietal region, suggestive of chronic hemorrhage. Early changes of cystic encephalomalacia noted.

Follow up cranial ultrasound findings on 12thday of life were suggestive of dilatation of bilateral lateral ventricles with 26 x $16 \mathrm{~mm}$ sized lesion with heterogenous echogenicity noted in left frontoparietal and basal ganglia region, suggestive of chronic intraparenchymal hemorrhage was observed.(Figure 2)

Plain CT scan of brain performed on $28^{\text {th }}$ day of life. Findings were suggestive of severe loss of Cerebral cortex, basal ganglia as well as white matter of cerebral cortex was seen in both cerebral hemisphere which was replaced by multiple cystic encephalomalacia changes. There was evidence of $25 \times 16.5$ $\mathrm{mm}$ sized heterogenous density area in left frontoparietal and basal ganglia region, suggestive of chronic hemorrhage with gross dilatation of both lateral ventricles noted.(Figure 3)

Plain MR imaging of brain performed at age of 2 months with high resolution $\mathrm{T} 1$ and $\mathrm{T} 2$ weighted serial sections obtained in the sagittal, coronal and axial planes on a $1.5 \mathrm{~T}$ scanner with high strength gradients. Findings werehypointense areas in left fronto-parietal region on SWI and appear hyperintense on T1 images suggestive of subacute hemorrhage.

On T2 weighted images multiple large hyperintense areas seen involving bilateral fronto-parieto-occipito-temporal regions almost completely replacing cerebral parenchyma which appear hypointense on T1W and FLAIR images, suggestive of changes of cystic encephalomalacia. Exvacuo dilatation of ventricles were also seen.(Figure 4).

\section{DISCUSSION}

Antenatal ultrasound allows an accurate diagnosis of fetal $\mathrm{ICH}$. The findings are variable and change with time. Acute cerebral hemorrhage appears as a brightly echogenic collection without posterior shadowing. Over the following days blood clots develop a complex echogenic texture with an external echogenic lining and an internal hypo to anechoic core. Intraventricular hemorrhage are usually associated with distension of the ventricles, which initially demonstrate a typical echogenic lining. Intraventricular hemorrhage may undergo spontaneous resolution or may obstruct the cerebrospinal fluid circulation, usually at the level of the aqueduct of Sylvius, resulting in ventriculomegaly. Involvement of the cortex can be predicted by demonstration of extension of the echogenic collection to the periventricular parenchyma in the early stages. Final outcome of this cerebral insult results in changes of encephalomalacia and gliosis.

In our study, antenatal ultrasound suggested intraparenchymal and intraventricular hemorrhage in fetal brain conformed on MRI, which progressively converted to chronic hemorrhage and changes of cystic encephalomalacia were observed about one month later as end result.

MRI has become a standard problem- solving tool in fetal imaging with utility in evaluation of brain and assessment of brain abnormalities. ${ }^{5,6}$

MRI findings of fetal $\mathrm{ICH}$ on $\mathrm{T} 1$ weighted images intraventricular clot or high signal parenchymal foci suggest vasculitis or petechial hemorrhage and on T2 weighted images low signal intraventricular clot, intraventricular fluidfluid level, abnormal cortical signal and porencephalic cyst formation. There is little information available regarding changes in signal of fetal intracranial blood products over time and is not typically used for follow up. If pregnancy continues, follow up imaging should be done with ultrasound. Live born infants may undergo serial CT or MRI scans as part of their management.

Encephalomalacia is an area of focal brain damage which when diffuse may present as large area of multisystem degeneration. The tendency of human brain to undergo dissolution and cavitation is a function of timing of insult. It had been described under a variety of synonyms in paediatric and pathological literature. Most cases show the lesions distributed in the region of anterior and middle cerebral arteries sparing cerebellum and medulla.

In our study, there was almost complete loss of cerebral cortex was found in later stage with sparing of cerebellum, pituitary gland and brainstem.

Amongst all the imaging modalities, cranial ultrasound is preferred as primary imaging modality of choice as well as for follow up in cystic encephalomalacia, apart from radiation protection due to

1. Diagnosis of the condition

2. Reliably assess the size of the cysts

Cranial ultrasound provide safe, cost effective, non invasive screening examination of hemodynamically unstable neonate at the bed side. Ultrasound within one week of the insult shows increased echogenicity in the affected areas with cystic degeneration appear 1-4 weeks later. Cranial ultrasound is the most sensitive modality for detection of glial septa but it lags behind MR in the overall brain evaluation.

Cranial ultrasound is also sensitive for detection of intracerebral hemorrhage, periventricular leukomalacia (PVL) and hydrocephalus. Ultrasound is operator dependent, however, and less sensitive to structural abnormalities in the cerebral convexities and in the brainstem. Parenchymal abnormalities, such as PVL and cerebral oedema, identified at US are also non specific. ${ }^{7}$

Gupta et al also emphasized that ultrasound is diagnostic for cystic encephalomalaia and does not warrant further investigation.

However Sanapo L, et al aimed to investigate the role of MRI in the diagnosis of fetal $\mathrm{ICH}$ among pregnancies reffered to fetal MRI and to characterize the topography of fetal $\mathrm{ICH}$ using MRI, when prenatal diagnosis of $\mathrm{ICH}$ was mainly 
based on US findings rather than MRI. They concluded that MRI is an important tool in prenatal diagnosis of $\mathrm{ICH}$, especially when US describes nonspecific intracranial abnormalities. $^{8}$

Differential diagnosis of cystic encephalomalacia include encephaloclastic porencephaly and hydrancephaly. The differentiation of an encephaloclastic porencephaly is differentiated from encephalomalacic cavity by its smooth walled, fluid filled cavity in communication with ventricular system while encephalomalacic cavity contains septations and irregular wall composed primarily of reactive astrocytes. Hydrancephaly is characterized by the destruction and resorption of most of the brain parenchyma and CSF filled thin walled cerebral hemispheres., ${ }^{2,3,4}$

In our study, we concluded that Ultrasound was almost diagnostic, and MRI, when performed, add further information. Because ultrasound has narrow window through anterior fontanelle, so visualized field is limited. MRI always proved accurate because multiplanar assessment of whole brain is possible with exceptionally detailed images. It also has the additional advantage that different sequences allow assessment of various pathologies and finally no ionizing radiation is required, so no radiation hazards. It is clear that there is certainly a role for this technology.

The diagnostic work-up of a pregnancy complicated by ultrasound evidence of an intracranial hemorrhage should include an interview focusing on maternal medicinal use or recent trauma (motor vehicle accident, fall onto the abdomen, others) and laboratory tests for the possible presence of a fetal platelet disorder. This should include maternal platelet count and testing for both maternal alloimmunity and isoimmunity. Antenatal hemorrhage is possible with isoimmune thrombocytopenia but is exceedingly rare. ${ }^{9}$

Whether treatment would be indicated in the presence of an established fetal intracranial lesion is uncertain. Severe lesions usually have a poor prognosis and conservative management may be offered to the patient.

\section{CONCLUSION}

Obstetric ultrasound screening should be considered in assessment of all pregnant patients for screening because it is safe, cost effective, easily available and can detect gross fetal congenital anomalies and early diagnosis of fetal and materal complications and helps in further management. In most cases, fetal MR allows for a confident diagnosis of fetal ICH. More detailed assessment of injured area may allow specific, targeted therapy to maximize functional outcome for affected children.

Cranium ultrasound should be considered in assessment and follow up of all neonates and infants who suffer from intracerebral hemorrhage, asphyxia and intracranial infection. However MRI is equally or even more helpful for accurate diagnosis as well as confirmation and assessment of fetal ICH and its sequel- cystic encephalomalacia, so MRI should also be considered in assessment of fetal ICH as detailed informative and confirmative modality.

As the prognosis of cystic encephalomalacia is grave so prompt recognition will facilitate timely counselling of parents. Hence cranial ultrasound is diagnostic as well as safe for follow up of the patient.

\section{REFERENCES}

1. Kutuk MS, Yikilmaz A, Ozgun MT, Dolanbay M, Canpolat M, Uludag $\mathrm{S}$ et al. Prenatal diagnosis and postnatal outcome of fetal intracranial hemorrhage. Childs Nerv Syst 2014; 30(1): 411-8.

2. Blankenberg FG, Loh NN, Bracci P, D'Arceuil HE, Rhine WD, Norbash AM, Lane B, Berg A, Person B, Coutant M, Enzmann DR. Sonography, CT, and MR imaging: a prospective comparison of neonates with suspected intracranial ischemia and hemorrhage. AJNR Am J Neuroradiol 2000;21(1):213-8.

3. Felderhoff-Mueser U, Rutherford MA, Squier WV, Cox P, Maalouf EF, Counsell SJ, Bydder GM, Edwards AD. Relationship between MR imaging and histopathologic findings of the brain in extremely sick preterm infants. AJNR Am J Neuroradiol 1999;20(7):1349-57.

4. Raybaud C. Destructive lesions of the brain. Neuroradiology 1983; 25(4): 265-291.

5. Rossi AC, Prefumo F. Additional value of fetal magnetic resonance imaging in the prenatal diagnosis of central nervous system anomalies: a systemic review of literature. Ultrasound Obstet Gynecol 2014; 44(5): 383-93.

6. Lyons K, Cassady C, Jones J, Palindo M, Mehollin-Ray A, Guimaraes C, et al. Current role of fetal magnetic resonance imaging in neurologic anomalies. Semin Ultrasound CT MR 2015; 36(6): 298-309.

7. Barkovich AJ.The encephalopathic neonate: Choosing the Proper Imaging Technique in American Journal of Neuroradiology 1997;18(4):1816-20.

8. Laura Sanapo et al. Fetal intracranial hemorrhage: role of fetal MRI. The Journal of Maternal-Fetal \& Neonatal Medicine 2017;32(2):21-30.

9. American College of Obstetricians and Gynecologists Women's Health Care Physicians. ACOG Practice Bulletin No.138: inherited thrombophilias in pregnancy. Obstet Gynecol 2013; 122(5): 706-17.

Source of Support: Nil; Conflict of Interest: None

Submitted: 21-10-2019; Accepted: 16-11-2019; Published online: 12-12-2019 